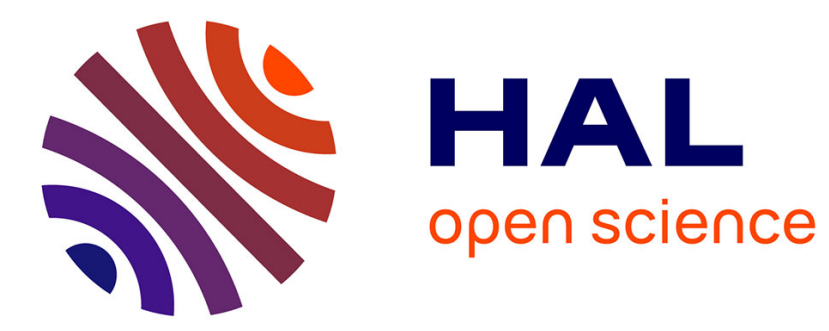

\title{
Learning the aircraft mass and thrust to improve the ground-based trajectory prediction of climbing flights
}

Richard Alligier, David Gianazza, Nicolas Durand

\section{To cite this version:}

Richard Alligier, David Gianazza, Nicolas Durand. Learning the aircraft mass and thrust to improve the ground-based trajectory prediction of climbing flights. Transportation research. Part C, Emerging technologies, 2013, 36, pp 45-60. 10.1016/j.trc.2013.08.006 . hal-00907651

\section{HAL Id: hal-00907651 \\ https://hal-enac.archives-ouvertes.fr/hal-00907651}

Submitted on 21 Nov 2013

HAL is a multi-disciplinary open access archive for the deposit and dissemination of scientific research documents, whether they are published or not. The documents may come from teaching and research institutions in France or abroad, or from public or private research centers.
L'archive ouverte pluridisciplinaire HAL, est destinée au dépôt et à la diffusion de documents scientifiques de niveau recherche, publiés ou non, émanant des établissements d'enseignement et de recherche français ou étrangers, des laboratoires publics ou privés. 


\title{
Learning the Aircraft Mass and Thrust to Improve the Ground-based Trajectory Prediction of Climbing Flights
}

\author{
R. Alligier ${ }^{\mathrm{a}, \mathrm{b}, *}$, D. Gianazza $\mathrm{a}, \mathrm{b}, * *$, N. Durand $\mathrm{d}^{\mathrm{a}, \mathrm{b}}$ \\ ${ }^{a}$ ENAC, MAIAA, F-31055 Toulouse, France \\ ${ }^{b}$ Univ. de Toulouse, F-31400 Toulouse, France
}

\begin{abstract}
Ground-based aircraft trajectory prediction is a major concern in air traffic control and management. A safe and efficient prediction is a prerequisite to the implementation of automated tools that detect and solve conflicts between trajectories. This paper focuses on the climb phase, because predictions are much less accurate in this phase than in the cruising phase.

Trajectory prediction usually relies on a point-mass model of the forces acting on the aircraft to predict the successive points of the future trajectory. The longitudinal acceleration and climb rate are determined by an equation relating the modeled power of the forces to the kinetic and potential energy rate. Using such a model requires knowledge of the aircraft state (mass, current thrust setting, position, velocity, etc.), atmospheric conditions (wind, temperature) and aircraft intent (thrust law, speed intent). Most of this information is not available to ground-based systems.

In this paper, we improve the trajectory prediction accuracy by learning some of the unknown point-mass model parameters from past observations. These unknown parameters, mass and thrust, are adjusted by fitting the modeled specific power to the observed energy rate. The thrust law is learned from historical data, and the mass is estimated on past trajectory points. The adjusted parameters are not meant to be exact, however they are designed so as to improve the energy rate prediction. The performances of the proposed method are compared with the results of standard modelbased methods relying on the Eurocontrol Base of Aircraft DAta (BADA), using two months of radar track records and weather data.
\end{abstract}

Keywords: aircraft trajectory prediction, BADA, energy rate, mass estimation, thrust law, Machine Learning 


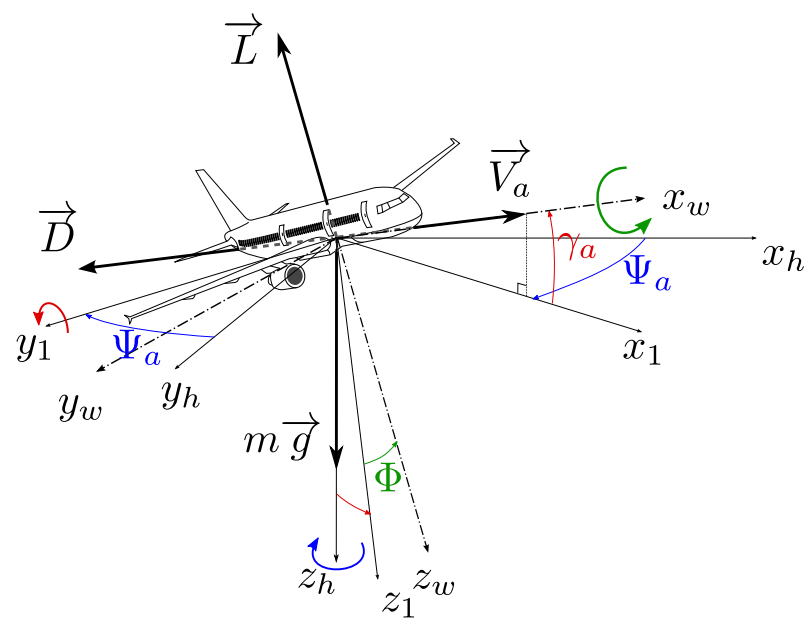

Figure 1: Euler rotations of angles $\left(\Psi_{a}, \gamma_{a}, \Phi\right)$ with counter-clockwise convention, and right-handed coordinate systems.

\section{Nomenclature}

$c \quad$ Thrust setting coefficient

CAS Calibrated Airspeed

$D \quad$ Drag

ESF Energy share factor

$g \quad$ Gravitational acceleration

$g_{0} \quad$ Gravitational acceleration at mean sea level

$h \quad$ Geodetic height

$H_{p} \quad$ Geopotential pressure altitude (i.e. geopotential altitude in ISA conditions)

ISA International Standard Atmosphere

$L \quad$ Lift

$M \quad$ Mach number

$m \quad$ Aircraft mass

$T \quad$ Air temperature

Thr Thrust

$V_{a} \quad$ True airspeed

$V_{a / x_{h} y}$ Projection of the true airspeed vector onto the local horizontal plane
$V_{g} \quad$ Ground speed (projection of the inertial speed on the local horizontal plane)

$V_{i} \quad$ Inertial velocity

$W \quad$ Wind, with $\left(W_{N}, W_{E}, W_{U p}\right)$ the Northbound, Eastbound, and upward components

$\gamma_{a} \quad$ Air-relative flight path angle

$\gamma_{i} \quad$ Inertial flight path angle (i.e. angle between $\vec{V}_{g}$ and $\vec{V}_{i}$ )

$\theta_{c} \quad$ Crab angle $\left(\Psi_{i}-\Psi_{a}\right)$

$\Phi \quad$ Bank angle

$\Psi_{a} \quad$ Aerodynamic heading (direction of true airspeed vector)

$\Psi_{i} \quad$ True course angle (direction of inertial velocity vector)

$\Psi_{W} \quad$ Direction of wind vector

$x_{k, i} \quad$ Vector of state variables (temperature differential, aircraft position, velocity, bank angle, etc.) for point $i$ in trajectory $k$

* Principal Corresponding Author

** Corresponding Author

Email addresses: richard.alligier@enac.fr(R. Alligier), 

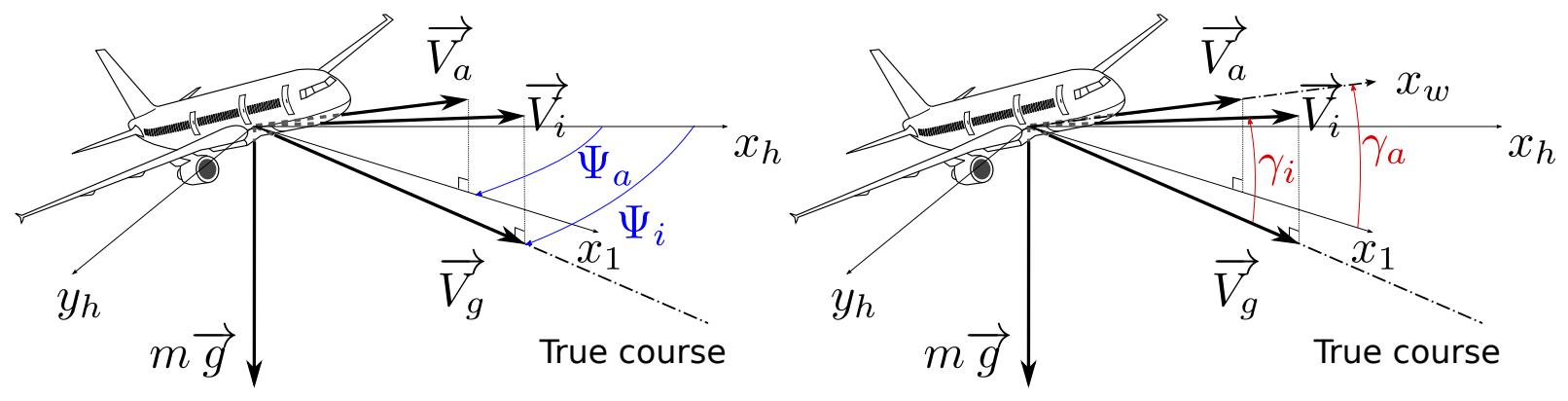

Figure 2: $\Psi$ and $\gamma$ angles (with $x_{h}$ pointing to the North)

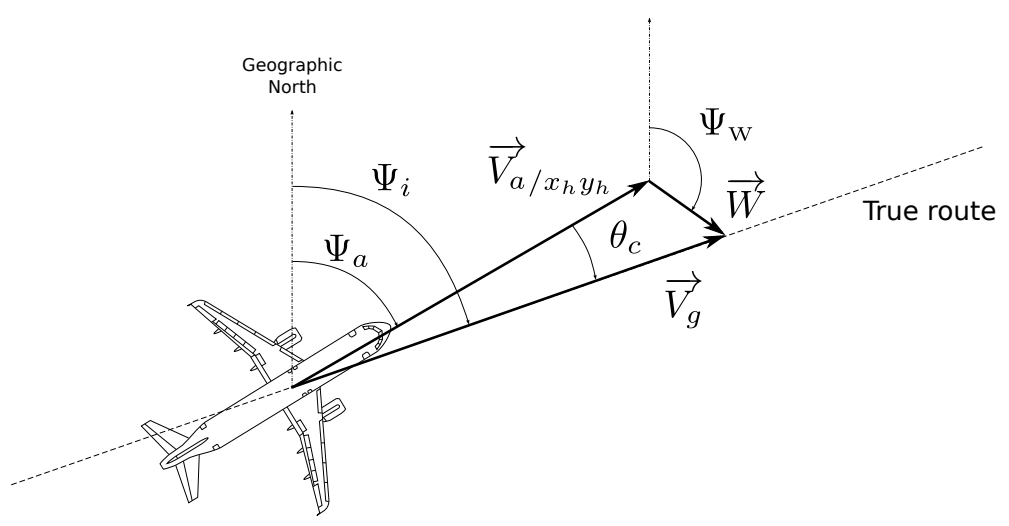

Figure 3: Angles in the local horizontal plane 


\section{Introduction}

Ground-based aircraft trajectory prediction is central to most applications in Air Traffic Control and Management (ATC/ATM) and has become more so with the emergence of new operational concepts [1, 2] envisioning trajectory-based operations. Moreover, trajectory prediction accuracy is essential to the new automated tools and algorithms that have emerged in the recent years. Some of these algorithms require to test a large number of alternative trajectories. As an example, in [3] an iterative quasiNewton method is used to find trajectories for departing aircraft, minimizing the noise annoyance. Another example is [4] where Monte Carlo simulations are used to estimate the risk of conflict between trajectories, in a stochastic environment. Some of the automated tools currently being developped for ATC/ATM can detect and solve conflicts between trajectories, using Genetic Algorithms ([5] $]^{1}$, or Differential Evolution or Particle Swarm Optimization ([7]). To be efficient, these methods require a fast and accurate trajectory prediction, and the capability to test a large number of "what-if" trajectories. Such requirements forbid the sole use of on-board trajectory prediction, which is certainly the most accurate, but is not sufficient for these most promising applications. So, even with the existing or future datalink capabilities that could transmit the on-board prediction to the ground systems, there remains a need for a fast and accurate ground-based prediction.

Unfortunately, ground-based trajectory predictors use point-mass models with unknown or uncertain inputs. Point-mass models relate the inertial acceleration to the forces acting on the center of mass. Such a model is formulated as a set of differential algebraic equations that must be integrated over a time interval in order to predict the successive aircraft positions, knowing the aircraft initial state (mass, current thrust setting, position, velocity, bank angle, etc.), atmospheric conditions (wind, temperature), and aircraft intent (thrust profile, speed profile, route, for example). Most of this information is either not available to ground systems, or known with uncertainty. In this paper, we focus on climb prediction, which is currently fairly inaccurate due to the basic assumptions currently being made on the input values of the point-mass model (e.g. : standard reference mass, reduced power climb at a given calibrated airspeed or Mach number).

Machine Learning is a paradigm of Artificial Intelligence, where a machine (e.g. a computer) extrapolates from a set of examples, so that it can make accurate predictions about future examples. Following this line, our approach is to learn some of the unknown point-mass model parameters from observations, in order to improve the trajectory prediction accuracy. Previous research studies have already followed this path, using probabilistic methods ([8]), online learning ([9]), or adaptive adjustments of some modeled parameters $([10,11,12,13])$. Considering a climbing aircraft, the past trajectory points are used to adjust either a modeled aircraft mass (assuming a

\footnotetext{
gianazza@recherche.enac.fr(D. Gianazza)

${ }^{1}$ These algorithms are at the root of the strategic deconfliction through speed adjustments developped in the European ERASMUS project ([6]). A more recent application is the SESAR 4.7.2 (Separation Task in En Route Trajectory-based Environment) project, where lateral and vertical maneuvers are also used.
} 
standard thrust profile), or a modeled thrust (assuming a standard reference mass) ${ }^{2}$ so as to minimize the error between the model and the observation. In [14], we introduce a Machine Learning approach where an optimal thrust law is learned from historical data, and where the individual aircraft masses are estimated from past trajectory points. The preliminary results show a good fit of the modeled power to the observed energy rate. In [15], the proposed mass estimation method is compared with the adaptive mechanism of Schultz et al. [13], showing better performances on simulated data.

In the present publication, we show how the thrust law learned from examples and the estimated mass enhance the altitude prediction when compared with the standard BADA predictor and several of its variants. Contrary to most previous studies that use only simulated data (or small samples of real data, for one of them), we validate the proposed method on a large amount of real data, using two months of recorded climbing trajectories from aircraft departing from the Paris-Orly and Paris-Charlesde-Gaulle airports. In addition, the mass estimation method is extended to the use of a BADA reduced climb power. An empirical assessment of the trajectory prediction quality is also given, considering how the model performs on the past trajectory.

The rest of the paper is organized as follows: Section 1 describes the models and equations that are useful for climb prediction. Section 2 introduces the analytical method used to estimate the aircraft mass, for a given thrust law. Section 3 describes how the optimal thrust law is learned from historical data. We show in section 4 how the inferred thrust law and estimated mass can be used for prediction purposes. The real data and experimental setup used in our experiments are detailed in section 5, and results are shown in section 6 . The last section concludes and gives some perspectives of future research.

\section{Models and Equations}

\subsection{The Second Newton's Law and Point-mass Model Equations}

Ground-based trajectory predictors for air traffic management and control usually rely on a simplified point-mass model to predict aircraft trajectories. In such a model, all forces acting on the aircraft body are exerted at the center of mass, making several simplifying approximations. The inertial moments and angular accelerations of the aircraft around its center of gravity are not included in the model. The aircraft is modeled as a point of mass $m$, subject to the second Newton's law that gives us the inertial acceleration $\overrightarrow{a_{i}}=\frac{d \vec{V}_{i}}{d t}=\dot{\vec{V}}_{i}$ of the center of mass (the dot above a vector denotes the time derivative of this vector):

$$
m \overrightarrow{V_{i}}=\overrightarrow{T h r}+\vec{D}+\vec{L}+m \vec{g}
$$

In equation (1), mass is considered a stationary variable ${ }^{3}$ regarding its impact on the aircraft dynamics. At a larger scale, however, the fuel burn and the consequent

\footnotetext{
${ }^{2}$ One of these studies $([\overline{8}])$ also models the wind as a stochastic variable.

${ }^{3}$ We assume in fact that $\frac{d}{d t}\left(m V_{i}\right)=m \dot{V}_{i}$, and neglect the impact of $\dot{m}$ on the acceleration.
} 
loss of mass must be taken into account when integrating the equations to predict the future trajectory. Concerning the forces, it is assumed that the thrust $\overrightarrow{T h r}$ exerted by the aircraft engines is aligned to the airspeed vector $\overrightarrow{V_{a}}$, and in the same direction. The drag $\vec{D}$ exerted by the relative wind on the flying airframe is also aligned to $\vec{V}_{a}$, by definition, and in the opposite direction. The lift force $\vec{L}$ caused by the motion of the airframe through the air is perpendicular to these vectors and in the plane of symmetry of the aircraft. The flight is assumed to be symmetric and there is no aerodynamic sideforce. The effects of Earth rotation on the aircraft dynamics are neglected (flat Earth approximation).

With the above simplifying approximations, and by expressing the forces in adequate coordinate systems, equation (1) can be transformed into the three following scalar equations governing the aircraft dynamics:

$$
\begin{aligned}
\dot{V}_{a}= & \frac{T h r-D}{m}-g \sin \gamma_{a}-\frac{\dot{\vec{W}} \cdot \vec{V}_{a}}{V_{a}} \\
\dot{\gamma}_{a}= & \frac{L \cos \Phi}{m V_{a}}-\frac{g \cos \gamma_{a}}{V_{a}} \\
& +\frac{1}{m V_{a}}\left[\dot{W}_{N} \sin \gamma_{a} \cos \Psi_{a}+\dot{W}_{E} \sin \gamma_{a} \sin \Psi_{a}-\dot{W}_{U p} \cos \gamma_{a}\right] \\
\dot{\Psi}_{i}= & \frac{L}{m V_{g}}\left(\sin \theta_{c} \sin \gamma_{a} \cos \Phi+\cos \theta_{c} \sin \Phi\right)-\left(\frac{T h r-D}{m V_{g}}\right) \sin \theta_{c} \cos \gamma_{a}
\end{aligned}
$$

\subsection{The Total Energy Model Equations, with the Effect of Wind}

Introducing the pressure altitude $H_{p}$ and a temperature correction that accounts for the use of the pressure altitude instead of the geodetic altitude, equation (2) can be written as follows:

$$
\underbrace{\frac{\text { Power }}{m}}_{\text {specific power }}=\underbrace{V_{a} \frac{d V_{a}}{d t}+g_{0}\left(\frac{T}{T-\Delta T}\right) \frac{d H_{p}}{d t}}_{\text {specific energy rate }}+\underbrace{\frac{d \vec{W}}{d t} \cdot \overrightarrow{V_{a}}}_{\text {wind effect }}
$$

In this equation, the vertical component of the wind is assumed to be zero because of the lack of sufficient information. In our data, the wind is known through a weather model updated every 3 hours. The vertical wind, as a local phenomenon due for example to convective weather, is out of the scope of such a weather model, which aims at providing wind data for Air Trafic Management purposes on a large scale. Such assumptions are most common in the literature dealing with similar subjects (see [16] for example). Note however that the effect of the wind gradient - i.e. the changes in direction and magnitude of the horizontal wind while moving along the vertical axis is taken into account. 
Equation (5) governs the energy rate of the aircraft, and relates both the longitudinal acceleration $\frac{d V_{a}}{d t}$ and the rate of climb $\frac{d H_{p}}{d t}$ to the power of the forces exerted at the center of mass (see [17] for more details). The power is defined as follows:

$$
\text { Power }=(T h r-D) V_{a}
$$

Considering the term representing the energy rate in the right-hand side of equation (5), we see that the available power that goes to the aircraft motion is shared between longitudinal acceleration $\frac{d V_{a}}{d t}$ and climb (or descent) rate $\frac{d H_{p}}{d t}$. The energy share factor ESF is defined as follows:

$$
E S F=\frac{g_{0}\left(\frac{T}{T-\Delta T}\right) \frac{d H_{p}}{d t}}{V_{a} \frac{d V_{a}}{d t}+g_{0}\left(\frac{T}{T-\Delta T}\right) \frac{d H_{p}}{d t}}=\left[1+\left(\frac{T-\Delta T}{T}\right)\left(\frac{V_{a}}{g_{0}}\right)\left(\frac{d V_{a}}{d H_{p}}\right)\right]^{-1}
$$

This energy share between longitudinal acceleration and rate of climb is directly related to how the aircraft is operated by the pilot (or the autopilot). Equation (7) does not come from a physical law as equation (5), but is just a definition of a quantity reflecting the pilot's choice, that is usually unknown to ground-based systems.

Equation (5) is sufficient when estimating the mass or learning the optimal thrust law from historical data, making no assumptions on the ESF law. However, if we want to predict the future pressure altitude $H_{p}$ and true airspeed $V_{a}$ of an aircraft, we do need to make some additional assumptions on the ESF law (constant CAS/Mach climb, or constant ROCD, for example).

\subsection{Computing the Aircraft Trajectory, Assuming Complete and Accurate Information}

Knowing the initial state $\left(H_{p}, V_{a}, m\right)$, the fuel flow given by the BADA model, and the two laws chosen for the thrust setting $c$ and energy share factor ESF, we can compute the variations of the state variables $H_{p}, V_{a}$ and $m$ over time, using equations (5) and (7).

Considering equation (5), the definition of the ESF given in equation (7) is completely equivalent to the following formulation:

$$
g_{0}\left(\frac{T}{T-\Delta T}\right) \frac{d H_{p}}{d t}=\left(\frac{\text { Power }}{m}-\frac{d \vec{W}}{d t} \cdot \overrightarrow{V_{a}}\right) \text { ESF }
$$

Denoting $\tau$ the ratio $\frac{T}{T-\Delta T}$, these equations can be reformulated into the differential algebraic equation 9 :

$$
\left(\begin{array}{cc}
\frac{1}{2} & g_{0} \tau \\
0 & g_{0} \tau
\end{array}\right)\left(\begin{array}{c}
\frac{d}{d t}\left(V_{a}^{2}\right) \\
\frac{d H_{p}}{d t}
\end{array}\right)=\left(\frac{\text { Power }}{m}-\frac{d \vec{W}}{d t} \cdot \overrightarrow{V_{a}}\right)\left(\begin{array}{c}
1 \\
E S F
\end{array}\right)
$$

When integrating equation (9) over time, we can compute the future altitude and speed of the aircraft as illustrated with the equation [10):

$$
\left(\begin{array}{c}
V_{a}^{2} \\
H_{p}
\end{array}\right)_{t}-\left(\begin{array}{c}
V_{a}^{2} \\
H_{p}
\end{array}\right)_{0}=\int_{0}^{t}\left(\frac{\text { Power }}{m}-\frac{d \vec{W}}{d t} \cdot \overrightarrow{V_{a}}\right)\left(\begin{array}{cc}
\frac{1}{2} & g_{0} \tau \\
0 & g_{0} \tau
\end{array}\right)^{-1}\left(\begin{array}{c}
1 \\
E S F
\end{array}\right) d t
$$


Equation (10) shows the key elements of aircraft trajectory prediction: mass, power, energy share factor, and weather (wind and air temperature). These are also the potential sources of uncertainty with which ground-based trajectory predictors have to deal.

\subsection{Model of the Forces and Power}

Computing a trajectory requires a computational model of the aerodynamic drag $D$ of the airframe flying through the air, and of the engines' thrust Thr. In our experiments, we used version 3.9 of the Eurocontrol BADA model (see [17]) to compute the drag $D$ and the maximum climb thrust $T h r_{\text {max climb }}$.

More accurate BADA models exist (BADA 4 family, [18]), and are available under license, for a smaller number of aircraft types. They actually model the aircraft dynamics more accurately than BADA 3.9 ([19]), fitting the manufacturer data better and making fewer simplifying assumptions. These enhancements are useful, especially for realistic simulations.

However, we are not actually looking for a more accurate point-mass model, here. Our aim is rather to improve the estimation of the input variables, which is a critical issue considering all the unknowns and uncertainties on these variables when using the point-mass model as a ground-based predictor. BADA 3.9 is sufficient for our purpose, in this context.

Considering the equations found in [17], $T h r_{\max c l i m b}$ is simply a function of $H_{p}, \Delta T$, and $V_{a}$, and the drag $D$ can be expressed as a polynomial of the mass $m$, which coefficients are functions of the other state variables:

$$
\begin{aligned}
& T h r_{\max c l i m b}=f_{1}\left(H_{p}, V_{a}, \Delta T\right) \\
& D=f_{2}\left(H_{p}, V_{a}, \Delta T\right)+m^{2} f_{3}\left(H_{p}, V_{a}, \Delta T, \Phi\right)
\end{aligned}
$$

As aircraft do not always fly at the maximum climb thrust in reality, we introduce a thrust reduction coefficient $c$, and model the thrust as follows:

$$
T h r=c T h r_{\max c l i m b}
$$

The power $(T h r-D) V_{a}$ can then be expressed as a function of the thrust setting coefficient $c$, the mass $m$, and the other state variables:

$$
\begin{aligned}
& \text { Power }=\left(c T h r_{\max \text { climb }}-D\right) V_{a} \\
& =\left(c f_{1}\left(H_{p}, V_{a}, \Delta T\right)-f_{2}\left(H_{p}, V_{a}, \Delta T\right)-m^{2} f_{3}\left(H_{p}, V_{a}, \Delta T, \Phi\right)\right) V_{a}
\end{aligned}
$$

At this point, it is important to notice that the power, given either by equation (13) or by the BADA equation for the reduced power climb ([17]), is a polynomial with respect to the mass $m$. This allows us to devise simple analytical methods to estimate the mass from the observations, as shown in the next section. 


\subsection{Modeling the thrust setting law $c=f(. \mid \theta)$}

The law governing the thrust setting $c$ can be modeled as a function $f(. \mid \theta)$ parametrized by $\theta=\left(\theta_{1}, \ldots, \theta_{q}\right)$, a vector of $q$ parameters. This law is assumed to be the same for all aicraft belonging to a given category. In the current paper, one such category contains all aircraft of a same type departing from a same airport. Further refinements may consider aircraft belonging to a same airline, or different categories for typical climb profiles extracted from the data.

Considering a set of trajectories with the same thrust setting law $f(. \mid \theta)$, the thrust coefficient at point $i$ of a trajectory $k$ is simply $c_{k, i}=f\left(x_{k, i} \mid \theta\right)$, with $x_{k, i}$ a vector of state variables (altitude $H_{p}$, velocity, bank angle, temperature differential $\Delta T$, for example) measured or computed at the $i^{\text {th }}$ point.

In order to learn the thrust law $f$ (see section 3 ) that gives the best prediction performances when used together with the estimated masses (see section 2), we need to choose a model for this law. This arbitrary choice may depend on the data made available to the ground-based predictor. As a proof of concept, we have chosen two models: one for the general case, and another one for the case of aircraft following a constant CAS/Mach climb, with the target CAS and Mach number known to the ground systems.

For the first model, used for Case Study 1 of section 6.3. we have chosen a polynomial function of the pressure altitude $H_{p}$ :

$$
f(x \mid \theta)=\sum_{i=0}^{4} \theta_{i}\left(H_{p}\right)^{i}
$$

For the second model, when considering CAS/Mach climbs with known target Calibrated Airspeed and Mach number, we have an opportunity to use this knowledge of the aircraft intent in the thrust law itself. This is done by introducing the crossover altitude $H_{p}^{\text {crossover }}$ in the polynomial function modeling the thrust setting. The following law is used in Case Study 2 of section 6.3

$$
f(x \mid \theta)=\sum_{i=0}^{4} \theta_{i}\left(H_{p}-H_{p}^{\text {crossover }}\left(C A S_{k}, M_{k}\right)\right)^{i}
$$

\section{Mass Estimation, Knowing the Thrust Setting}

Using equation (5), the mass $m$ can be estimated in a very simple way for a given thrust setting $c$. The specific power is a polynomial with respect to the mass $m$, and

we can measure the energy rate $V_{a} \cdot \frac{d V_{a}}{d t}+g \cdot \frac{d h}{d t}$ and wind effect $\frac{d \vec{W}}{d t} \cdot \vec{V}_{a}$ from recorded weather and radar data. Note that the mass can be estimated without making any assumption on the ESF law. 


\subsection{Equivalent Mass at a Given Point}

Introducing the total energy Energy $=\frac{1}{2} m V_{a}^{2}+m g h$ and considering equation (5), let us introduce $P$ and $Q$, defined as follows:

$$
\begin{aligned}
& P=\text { Power }-m \underbrace{\left[\frac{d}{d t}\left(\frac{\text { Energy }}{m}\right)+\left(\frac{d \vec{W}}{d t} \cdot \overrightarrow{V_{a}}\right)\right]}_{Q} \\
& Q=V_{a} \frac{d V_{a}}{d t}+g_{0}\left(\frac{T}{T-\Delta T}\right) \frac{d H_{p}}{d t}+\frac{d \vec{W}}{d t} \cdot \vec{V}_{a}
\end{aligned}
$$

The quantity $Q$ can be computed at any point of the past trajectory, or at any point of a recorded trajectory, using the available radar and weather information. Considering the power model given by equation (13) in section 1.2, we see that $P$ is a simple polynomial function of the second degree with respect to the aircraft mass $m$, when the thrust setting $c$ is fixed. So the total energy model equation (5) becomes:

$$
\frac{P(m)}{m}=0
$$

with

$$
\begin{aligned}
P(m)= & -m^{2} f_{3}\left(H_{p}, V_{a}, \Delta T, \Phi\right)-m Q \\
& +c f_{1}\left(H_{p}, V_{a}, \Delta T\right)-f_{2}\left(H_{p}, V_{a}, \Delta T\right)
\end{aligned}
$$

These equations are valid at any given point $i$ of the past trajectory, knowing $V_{a}$, $H_{p}, \Delta T$ and the thrust setting $c$. The equivalent mass $m_{i}$ at point $i$ is obtained by solving equation (17), giving us two possible solutions. In our experiments, only one solution was positive.

\subsection{Least Squares Estimation of the Mass, Using Several Points}

With the one-point method presented in the previous section, we observed on a few recorded trajectories that the estimated mass changes from one point to the next. In order to avoid the variations that cannot be explained solely by the fuel consumption or by a change in the thrust setting, we now introduce a least-squares approximation of the mass, using several points instead of only one.

Let us consider $n$ points in a given climb segment, with different values of the thrust settings $C=\left(c_{1}, \ldots, c_{n}\right)$. The mass is assumed to be constant over the considered segment. This approximation is reasonable: When simulating the climb of an Airbus A320 from FL130 to FL300 with the BADA model, in ISA+20 atmospheric condition, we find a fuel consumption of $1,075 \mathrm{~kg}$, that is to say $1.68 \%$ of the initial mass, chosen as the BADA reference mass.

Note that we do not try to adjust the thrust coefficients $C=\left(c_{1}, \ldots, c_{n}\right)$ here. Assuming a constant mass $m$ for the $n$ considered points, we adjust $m$ so as to minimize the following mean square error, given the thrust settings $\left(c_{1}, \ldots, c_{n}\right)$ :

$$
\mathcal{E}_{\text {traj }}(C, m)=\frac{1}{n} \sum_{i=1}^{n}\left(\frac{\text { Power }_{i}(m)}{m}-Q_{i}\right)^{2}
$$


Denoting $P_{i}(m)$ the value at point $i$ of the polynomial expression $P$ given by equation (17), the error becomes:

$$
\mathcal{E}_{t r a j}(C, m)=\frac{1}{n} \sum_{i=1}^{n}\left(\frac{P_{i}(m)}{m}\right)^{2}
$$

The minimum of $\mathcal{E}_{t r a j}$ must satisfy $\frac{d \mathcal{E}_{t r a j}}{d m}=0$, which gives us the following equation, with $P_{i}^{\prime}(m)$ the derivative of $P_{i}(m)$ with respect to $m$ :

$$
\sum_{i=1}^{n} P_{i}(m)\left[m P_{i}^{\prime}(m)-P_{i}(m)\right]=0
$$

This fourth-degree polynomial equation (21) is solved using Ferrari's method. Among the four potential solutions, we select the solution in $] 0 ;+\infty\left[\right.$ minimizing $\mathcal{E}_{t r a j}(C, m)$.

\subsection{Equivalent Mass in the Case of a BADA Reduced Climb Power}

We have seen how to estimate the mass at a given point (section 2.1) or using several trajectory points (section 2.2) when the power is modeled as described in equation (13). These methods can still be applied when assuming the BADA maximum climb, by setting $c=1$ in equation (18).

When considering the BADA reduced climb power, however, the expression of $P(m)$ is no longer the same. In this case, the power is given by Power $_{\mathrm{BADA}}$, described in the following equation [22), where $C_{\text {pow, red }}$ is the BADA power reduction coefficient depending on the mass and the maximum altitude $H_{\max }$ (see [17]):

$$
\text { Power }_{\mathrm{BADA}}=C_{\text {pow, red }}\left(T h r_{\text {max climb }}-D\right) V_{a}
$$

Using the BADA power reduction profile, we have the following equation for $P(m)$, which is still a polynomial function of the mass, as in section 2.1. but of the $3^{\text {rd }}$ degree below $0.8 H_{\max }$, and of the $2^{\text {nd }}$ degree above:

$$
\begin{aligned}
P(m)= & \text { Power }_{\mathrm{BADA}}-m Q \\
= & C_{\mathrm{pow}, \text { red }}(m)\left[-m^{2} f_{3}\left(H_{p}, V_{a}, \Delta T, \Phi\right)-f_{2}\left(H_{p}, V_{a}, \Delta T\right)+f_{1}\left(H_{p}, V_{a}, \Delta T\right)\right] \\
& \quad-m Q
\end{aligned}
$$

The equivalent mass can still be found by solving the polynomial equation 21, except that $P_{i}(m)$ is of the $2^{\text {nd }}$ or $3^{r d}$ degree, depending on the altitude of the considered points. In our experiments, when estimating the equivalent mass of an Airbus A320 using points below $18,000 \mathrm{ft}$ and assuming a BADA reduced climb power, the inequality $H_{p} \leq 0.8 H_{\max }(\Delta T, m)$ can reasonably be assumed to be true $e^{4}$ In that case, $P_{i}(m)$ in equation 21$]$ is a third-degree polynomial.

In any case, we have a simple method to estimate an equivalent mass from a few points of the trajectory, given the thrust law for these points.

${ }^{4}$ Numerical application sets $0.8 H_{\max }$ at $19,144 \mathrm{ft}$ for $\Delta T$ at $40 K$ and $m$ at $77,000 \mathrm{~kg}$. 


\section{Learning the Thrust Law from Historical Data}

We have seen in section 2 how to estimate the mass. Now, let us see how to learn the thrust setting law, using a set of recorded trajectories and a regression method coupled to our mass estimation method.

\subsection{Discussion}

We cannot infer both the mass and thrust setting law from equation (5) using only the past trajectory of one given aircraft: Denoting $C_{\text {past }}=\left(c_{1}, \ldots, c_{n}\right)$ the thrust coefficient for the past $n$ points, there is an infinity of couples $\left(C_{\text {past }}, m\right)$ fitting the observed energy rate perfectly. Intuitively, high thrust with a large mass $m$ barely produces the same amount of specific power $\frac{\left(c T h r_{\text {max climb }}-D\right) V_{a}}{m}$ as low thrust with a small mass $m$.

In a prediction context, we need to compute the specific power for the next $p$ points, which requires some assumptions on the future thrust settings $\left(c_{n+1}, \ldots, c_{n+p}\right)$. As this data is not available to ground systems, it is usual to make an "educated guess", assuming maximum climb thrust or reduced power climb for example, and to estimate an equivalent mass that is consistent with this choice, using the first trajectory points. As an alternative to this method, we propose to learn an optimal thrust law from historical data, choosing the law that minimizes the prediction error on a set of recorded trajectories.

\subsection{Learning a Thrust Law from Data}

We are looking for a thrust setting law $c=f(. \mid \theta)$ that is common to all aircraft of a same category. Given this law, the thrust setting coefficients $C_{k}=\left(c_{k, 1}, \ldots, c_{k, n_{k}}\right)$ for the $n_{k}$ points of a sampled trajectory $k$ are given by the following expression [24], with $x_{k, i}$ a vector of state variables measured at the $i^{t h}$ point:

$$
C_{k}(\theta)=\left(f\left(x_{k, 1} \mid \theta\right), \ldots, f\left(x_{k, n_{k}} \mid \theta\right)\right)
$$

Considering a set of $K$ trajectories taken from historical data, we are looking for a value of $\theta$ that minimizes the overall quadratic error $\mathcal{E}_{\text {total }}$, as defined by the following equations 25):

$$
\begin{aligned}
& m_{k}^{*}(\theta)=\underset{\left.m_{k} \in\right] 0 ;+\infty[}{\operatorname{argmin}} \mathcal{E}_{k}\left(C_{k}(\theta), m_{k}\right) \\
& \mathcal{E}_{\text {total }}(\theta)=\sum_{k=1}^{K} \mathcal{E}_{k}\left(C_{k}(\theta), m_{k}^{*}(\theta)\right)
\end{aligned}
$$

The optimal mass $m^{*}$ for any given $\theta$ is obtained by solving a polynomial equation using Ferrari's method, as presented in section 2.2.

In equations $(25), \mathcal{E}_{k}$ is the quadratic error for trajectory $k$, as given by equations 19 and 20) (see section 2.2). To be more explicit we can write $\mathcal{E}_{k}$ as follows: 


$$
\begin{aligned}
& \mathcal{E}_{k}\left(C_{k}, m_{k}\right)=\frac{1}{n_{k}} \sum_{i=1}^{n_{k}}\left(\frac{P_{k, i}\left(c_{k, i}, m_{k}\right)}{m_{k}}\right)^{2} \\
& P_{k, i}\left(c_{k, i}, m_{k}\right)=\operatorname{Power}_{k, i}\left(m_{k}\right)-m_{k} Q_{k, i} \\
& \operatorname{Power~}, i_{k}\left(m_{k}\right)=\left(c_{k, i} \text { Thr }_{\text {maxclimb }, i}-D_{k, i}\right) V_{a k, i}
\end{aligned}
$$

with $Q_{k, i}$ the value at point $i$ of trajectory $k$ of the quantity $Q$, the sum of the specific energy rate and wind effect, defined by equation (16b). The maximum climb thrust $T h r_{\text {maxclimb }}$ is given by the BADA equations as a function of the vector of state variables $x_{k, i}$. The drag $D$ is also provided by the BADA model, as a function of $x_{k, i}$ and $m_{k}$.

An iterative quasi-Newton method (BFGS) is used to find a vector $\theta^{*}$ minimizing $\mathcal{E}_{\text {total }}(\theta)$. The BFGS method (Broyden-Fletcher-Goldfarb-Shanno, from the names of its authors) approximates the Hessian matrix at each iteration, using the gradient of the error function $\nabla \mathcal{E}_{\text {total }}$. The reader may refer to [20] for further details on the method.

The gradient of the error can easily be computed in our problem. The partial derivative of the error on trajectory $k$ with respect to one parameter $\theta_{j}$ of vector $\theta$ is the following, considering that $\frac{\partial \mathcal{E}_{k}}{\partial m}\left(C_{k}, m_{k}^{*}\right)=0$, by definition of $m_{k}^{*}$ :

$$
\begin{aligned}
\frac{\partial \mathcal{E}_{k}}{\partial \theta_{j}}(\theta) & =\frac{\partial \mathcal{E}_{k}}{\partial m}\left(C_{k}, m_{k}^{*}\right) \frac{\partial m_{k}^{*}}{\partial \theta_{j}}+\sum_{i=1}^{n_{k}} \frac{\partial \mathcal{E}_{k}}{\partial c_{k, i}}\left(C_{k}, m_{k}^{*}\right) \frac{\partial c_{k, i}}{\partial \theta_{j}} \\
& =\sum_{i=1}^{n_{k}} \frac{\partial \mathcal{E}_{k}}{\partial c_{k, i}}\left(C_{k}, m_{k}^{*}\right) \frac{\partial f_{k, i}\left(x_{k, i} \mid \theta\right)}{\partial \theta_{j}}
\end{aligned}
$$

Using the equations 26a defining $\mathcal{E}_{k}$ and the above equation (27), we find the following expression for the partial derivative:

$$
\frac{\partial \mathcal{E}_{k}}{\partial \theta_{j}}(\theta)=\frac{2}{n_{k}} \sum_{i=1}^{n_{k}}\left(\frac{P_{k, i}\left(m_{k}^{*}\right)}{m_{k}^{*}} \frac{V_{a k, i} T h r_{k, i}}{m_{k}^{*}} \frac{\partial f\left(x_{k, i} \mid \theta\right)}{\partial \theta_{j}}\right)
$$

Finally, the components of the overall gradient vector $\nabla \mathcal{E}_{\text {total }}$ are the following:

$$
\frac{\partial \mathcal{E}_{\text {total }}}{\partial \theta_{j}}(\theta)=\sum_{k=1}^{K} \frac{\partial \mathcal{E}_{k}}{\partial \theta_{j}}(\theta)
$$

Equations (25) and (29) are applied on a set of recorded trajectories to compute the total error and its gradient. Using these two functions of $\theta$, the BFGS method finds the vector of adjusted parameters $\theta^{*}$ minimizing $\mathcal{E}_{\text {total }}(\theta)$. At each step of the BFGS search, the mass estimation method presented in section 2.2 is used to find the optimal masses $\left(m_{1}^{*}(\theta), \ldots, m_{K}^{*}(\theta)\right)$ corresponding to the candidate vector $\theta$ being evaluated. At the end of the search, we have the optimal $\theta^{*}$ and the optimal masses $\left(m_{1}^{*}\left(\theta^{*}\right), \ldots, m_{K}^{*}\left(\theta^{*}\right)\right)$ for the chosen trajectory set.

The learning phase described above provides us with a thrust setting law $f\left(. \mid \theta^{*}\right)$ which can then be used for prediction purposes on fresh trajectories. If the sample of 
historical data used for learning is sufficiently representative of the actual traffic (as we hope it is), the proposed method will generalize well when applied to prediction. The learned thrust law may not actually reflect the actual thrust settings of each individual aircraft, but it is designed so as to improve the predicting performance when coupled with the equivalent mass estimation method described in section 2.2 .

The choice of the parametric function $f$ for the thrust law is not discussed here. We have chosen a simple fourth-degree polynomial function of the pressure altitude $H_{p}$, as a proof of concept. Experimenting different choices for $f$ could be the subject of future works.

\section{Using the Inferred Mass and Thrust Law for Prediction Purposes}

Predicting the future trajectory of a climbing aircraft with the mass estimation method presented in section 2.2 and the thrust law learned from historical data is quite straightforward. Let us assume that we have observed $n$ points of the past trajectory, with one point every $\delta t$ seconds, and that we want to predict the next $p$ points of the future trajectory.

The thrust setting coefficients $c_{1}, \ldots, c_{n}$ for the past $n$ points are computed using the thrust law $f\left(. \mid \theta^{*}\right)$ learned from historical data (see section 3.2). Knowing the values

of the state variables $x_{i}^{(o b s)}$ (air temperature, aircraft altitude, velocity, etc.) for the past trajectory, the coefficients are simply $c_{i}=f\left(x_{i}^{(o b s)} \mid \theta^{*}\right)$, for all $i \in\{1, \ldots, n\}$. The equivalent mass $m$ is then estimated from the past trajectory, using these thrust setting coefficients and the method presented in section 2.2

Computing the thrust settings $\left(c_{n+1}, \ldots, c_{n+p}\right)$ for the next $p$ points is more difficult, as we need the predicted values of the state variables $\left(x_{n+1}^{(\text {pred })}(m), \ldots, x_{n+p}^{(\text {pred })}(m)\right)$. This requires to integrate equation 90 over the time interval $\left[t_{n}, t_{n+p}\right]$. Equation (10) shows the mathematical form of this integral of the ESF and specific power. In general, this equation cannot be solved analytically, and numerical methods must be used. Using for example the Euler method with a time step $\delta t$ (or Runge-Kutta methods), we can compute the successive values of the predicted state variables and thrust setting coefficients.

\section{Data and Experimental Setup}

\subsection{Data Pre-processing}

Recorded radar tracks from Paris Air Traffic Control Center are used in this study. This raw data is made of one position report every 1 to 3 seconds, over two months (July 2006, and January 2007). In addition, the wind and temperature data from Météo France are available at various isobar altitudes over the same two months.

The pressure altitude is reported by increments of $100 \mathrm{ft}([21])$ by the Mode-C radars. A local quadratic model is used to smooth our raw data. After smoothing, the basic trajectory data is made of the following fields: aircraft position $(X, Y$ in a projection plan, or latitude and longitude in WGS84), ground velocity vector $V_{g}=$ $\left(V_{x}, V_{y}\right)$, smoothed altitude $\left(H_{p}\right.$, in feet above isobar $\left.1,013.25 \mathrm{hPa}\right)$, rate of climb or 
descent $\frac{d H_{p}}{d t}$. The wind $W=\left(W_{x}, W_{y}\right)$ and temperature $T$ at every trajectory point are interpolated from the weather datagrid. The temperature differential $\Delta T$, which is the difference between the actual temperature and the temperature given by the ISA model, is computed at each point. This $\Delta T$ is one of the key parameters in the BADA model equations.

Using the position, velocity and wind data, we compute the true airspeed $V_{a}$, the distance flown in the air, the drift angle, the along-track and cross-track winds ( $W_{\text {along }}$ and $\left.W_{\text {cross }}\right)$. The successive velocity vectors allow us to compute the trajectory curvature at each point. The aircraft bank angle is then derived from true airspeed and the curvature of the air trajectory. The climb, cruise, and descent segments are then identified, using triggers on the rate of climb or descent to detect the transitions between two segments.

\subsection{Filtering and Sampling Climb Segments}

To demonstrate the performance of the proposed method, we focus on a single aircraft type (Airbus A320). Our dataset comprises all flights of this type departing from Paris-Orly (LFPO) or Paris-Charles de Gaulle Airport (LFPG). Of course, this approach can be replicated to other aircraft types and airports, and to more specific categories of climbs (e.g. per airline, per departure procedure, etc.) provided sufficient data can be collected.

The trajectories are filtered so as to keep only the climb segments. An additional 80 seconds is clipped from the beginning and end of each segment, so as to remove climb/cruise or cruise/climb transitions.

The trajectories are then sampled every 15 seconds so that the time $t_{0}$ of the "current" position corresponds to the crossing of altitude $18,000 \mathrm{ft}$. The 10 points before $t_{0}$ represent the "past trajectory", below FL180. The 40 points after $t_{0}$ represent the "future" trajectory (10 minutes ahead $t_{0}$ ). The first 11 points (current point and past trajectory) are used to estimate the equivalent mass. The remaining points (future trajectory) are used to compute the error between the predicted and actual energy rate.

\subsection{Two Case Studies, and Cross-validation}

As we have seen in section 1.3 , predicting the future trajectory does require some knowledge of the law governing the Energy Share Factor $(E S F)$. Learning the ESF law is not the subject of the work presented here, so in the following, we will either assume that the ESF is perfectly known, or make basic assumptions on the ESF law.

In our experiments, the prediction error of the proposed method is evaluated on two datasets, with two ways to obtain the ESF law. In the first case study, we use all the trajectories of our dataset (5,167 trajectories), and we look at the error on the energy rate prediction, assuming that the $E S F$ law is perfectly known. In the second case study, we assume a CAS/Mach climb to compute the $E S F$, and evaluate the overall performance of the trajectory prediction. In this case, as we have only Mode-C, and not Mode-S radar data that would contain the Calibrated Airspeed (CAS) and Mach number, we simply select the trajectories exhibiting a consistent CAS/Mach profile from our initial dataset. 
In both case studies, a ten-fold cross-validation is performed. The set of trajectories is split in ten folds of equal size. Nine folds are grouped together and used as the training set in order to learn the thrust law $f\left(. \mid \theta^{*}\right)$. The remaining fold is used as a validation set, to evaluate the prediction performance. The operation is repeated ten times, choosing a different fold as the validation set, in order to cover all possible combinations of couples (training set, validation set).

\subsection{Different Settings Compared}

Our prediction method uses one estimated mass $m_{\text {estimated }}$ per individual aircraft (see section 2.2) and a common thrust law $f\left(. \mid \theta^{*}\right)$ learned on the training set (see section 3.2. In order to evaluate the performance of the proposed method, it is compared with other model-based prediction methods relying on the BADA model. The BADA methods use a single reference mass for all aircraft of the same type, and either the BADA maximum climb thrust $\left(B A D A_{\max }\right.$ climb $)$ or the BADA power reduction profile $\left(B A D A_{\text {reduced }}\right)$, as given by equation 22].

The effects of mass estimation and thrust/power law can be evaluated independently by considering all possible combinations of mass and thrust/power law. The possible choices for the law governing the thrust (or the power for $B A D A_{\text {reduced }}$ ) during climb are summarized in table 1 .

\begin{tabular}{|c|c|}
\hline Name & Description \\
\hline \hline$B A D A_{\text {max climb }}$ & Power $=\left(T h r_{\max \text { climb }}-D\right) V_{a}$ \\
\hline$B A D A_{\text {reduced }}$ & $\begin{array}{c}\text { Power }=C_{\text {pow, red }}\left(T h r_{\text {max climb }}-D\right) V_{a} \\
C_{\text {pow, red }} \text { is the power reduction coefficient given by [17] }\end{array}$ \\
\hline Thrust law $f\left(. \mid \theta^{*}\right)$ & $\begin{array}{c}\text { Power }=\left(f\left(x \mid \theta^{*}\right) T h r_{\text {max climb }}-D\right) V_{a} \\
f\left(. \mid \theta^{*}\right) \text { is the thrust law learned on the training set (see section 3.2 }\end{array}$ \\
\hline
\end{tabular}

Table 1: Possible laws governing the thrust or power during climb

Concerning the mass, we could just compare the performances given by the estimated mass $m_{\text {estimated }}$ with the performances obtained when using the reference BADA mass $m_{B A D A}$, which is the usual way to proceed. To be more fair, we have also compared with another mass $m_{o p t}$, also common to all aircraft but chosen so as to minimize the altitude prediction error on the training set. The mass $m_{\text {opt }}$ minimizes the root mean square error on the pressure altitude $H_{p_{(51)}}$ at the last trajectory point, considering all trajectories in the training set. The possible choices for the mass setting are summarized in table 2 .

In our experiments, we compare our method combining $m_{\text {estimated }}$ and the learned thrust law $f\left(. \mid \theta^{*}\right)$ with the six different methods obtained by combining a mass $\left(m_{B A D A}\right.$, $m_{\text {opt }}$, or $m_{\text {estimated }}$ ) and a standard thrust/power law (either $B A D A_{\text {max climb }}$ or $B A D A_{\text {reduced }}$ ). The performances of the seven methods are assessed on the two case studies described in section 5.3, applying a ten-fold cross-validation. Section 6.3 shows the results (mean value and standard deviation) over the ten runs. Before comparing the seven methods, let us see a few results on the distribution of the estimated masses, and on the learned thrust law. 


\begin{tabular}{|c|c|}
\hline Name & Description \\
\hline$m_{B A D A}$ & $m=m_{B A D A}(A 320)=64,000 \mathrm{~kg}$ \\
\hline$m_{o p t}$ & $m=\underset{m \in] 0 ;+\infty[}{\operatorname{argmin}} \sum_{k=1}^{K}\left(H_{p(51)}^{(\text {obs })}-H_{p(51)}^{(\text {pred })}(m)\right)^{2}$ \\
\hline$m_{\text {estimated }}$ & $m_{k}=\underset{m \in] 0 ;+\infty[}{\operatorname{argmin}} \sum_{i=1}^{11}\left(\frac{P_{k, i}(m)}{m}\right)^{2}$ \\
\hline
\end{tabular}

Table 2: Possible choices for the aircraft mass

\section{Results}

\subsection{Estimated mass}

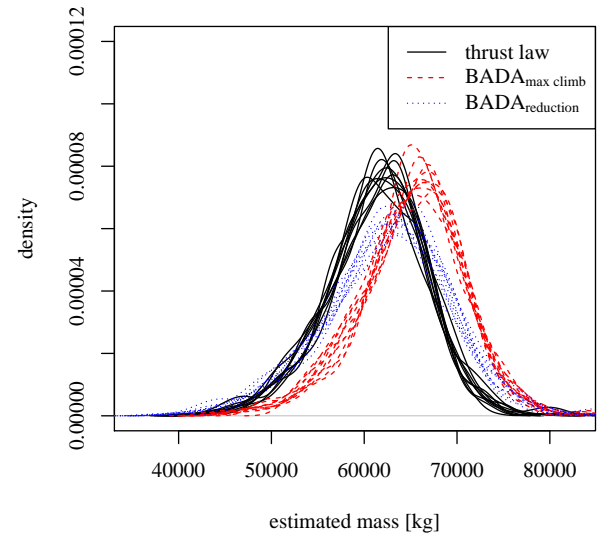

(a) Case study 1, all the trajectories

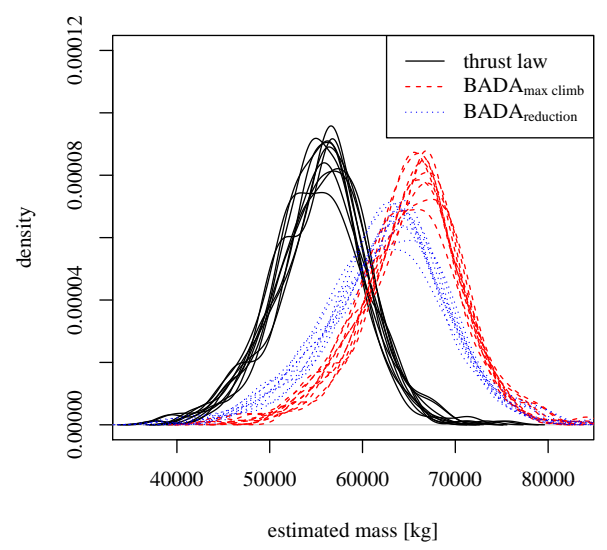

(b) Case study 2, CAS/Mach trajectories

Figure 4: Distributions of $m_{\text {estimated }}$ for the three thrust profiles (BADA max climb $\left.B A D A_{\text {reduced }}, f\left(. \mid \theta^{*}\right)\right)$ and for the 10 training sets of the cross-validation.

Figure 4 plots the distributions of the estimated masses for the two case studies, and for the three possible laws governing the thrust (or the power in the case of $B A D A_{\text {reduced }}$ ) during the climb. We have plotted 10 curves per thrust law, corresponding to the 10 training sets of the cross-validation.

We can see on this figure that the curves of a same thrust law are quite similar. The range of the estimated masses seems quite large for all distributions. The mean values differ from one law to another, which was to be expected as different laws lead to different thrust settings (or reduced power) coefficients and different masses: the higher the thrust setting, the higher the estimated mass.

We cannot compare the estimated masses with the actual aircraft masses, as the latter are not available to us. Therefore, we cannot say which combination of mass 
estimation method and thrust law provides the most realistic mass distribution. This is not an issue for our problem, however, as we estimate an equivalent mass meant to provide an accurate computation of the specific power, when associated with the adequate thrust law.

\subsection{Thrust law}

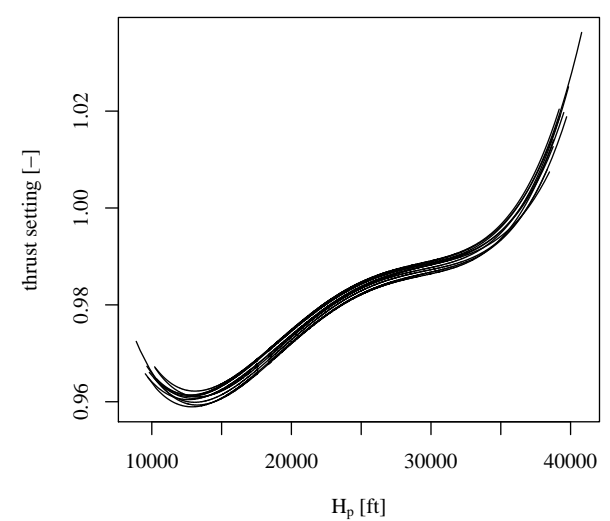

(a) Case study 1, all the trajectories

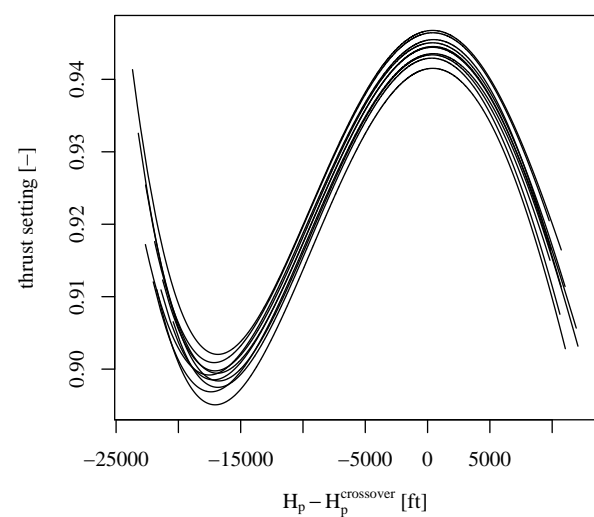

(b) Case study 2, CAS/Mach trajectories

Figure 5: The learned thrust law $f\left(. \mid \theta^{*}\right)$, for the 10 training sets of the cross-validation.

Figure 5 displays the thrust laws learned on the ten training sets while performing the cross-validation. The thrust profile is very similar for all the training sets. For the case study with all the trajectories (left picture), the thrust setting globally increases with the pressure altitude $H_{p}$ from 0.96 to 1.04 . For the CAS/Mach trajectories (right picture), the thrust setting oscillates between 0.90 and 0.95 , with a noticeable peak at the CAS/Mach crossover altitude.

\subsection{Overall Prediction Performance}

For a given trajectory, the prediction performance is evaluated by measuring the error between the predicted and observed values at equal times, for the $p$ points of the future trajectory. More explicitly, the prediction error over the $p$ future points of a given trajectory $k$ is assessed by measuring $e_{k}^{(p r e d)}=\sqrt{\mathcal{E}_{k}}$, with $\mathcal{E}_{k}$ given by equation 26, using the predicted state variables to compute the specific power. This quantity can be written as follows:

$$
e_{k}^{(\text {pred })}=\sqrt{\frac{1}{p} \sum_{i=1}^{p}(\underbrace{\frac{\text { Power }\left(m_{k}^{*}, c_{k, i}^{(\text {pred })}\right)}{m_{k}^{*}}}_{\text {computed }}-\underbrace{Q\left(x_{k, i}^{(o b s)}\right)}_{\text {observed at point } i})^{2}}
$$




\begin{tabular}{|c|c||c|c||c|c|}
\hline Thrust/power law & Mass & $e_{k}^{(\text {pred })}$ & $\mathrm{p}$-value & $\Delta H_{p}$ & $\Delta V_{a}$ \\
\hline$B A D A_{\text {max climb }}$ & $m_{B A D A}$ & $10.5(0.26)$ & $1.78 \mathrm{e}-227$ & $1223(41)$ & $6.51(0.52)$ \\
\hline$B A D A_{\text {max climb }}$ & $m_{\text {opt }}$ & $10.5(0.29)$ & $4.65 \mathrm{e}-234$ & $1203(46)$ & $6.61(0.57)$ \\
\hline$B A D A_{\text {max climb }}$ & $m_{\text {estimated }}$ & $9.2(0.4)$ & $9.71 \mathrm{e}-04$ & $895(60)$ & $6.02(0.35)$ \\
\hline$B A D A_{\text {reduced }}$ & $m_{B A D A}$ & $11.1(0.23)$ & $4.2 \mathrm{e}-280$ & $1352(37)$ & $6.91(0.39)$ \\
\hline$B A D A_{\text {reduced }}$ & $m_{\text {opt }}$ & $10.5(0.28)$ & $7.57 \mathrm{e}-219$ & $1198(45)$ & $6.63(0.54)$ \\
\hline$B A D A_{\text {reduced }}$ & $m_{\text {estimated }}$ & $9.26(0.4)$ & $3.35 \mathrm{e}-96$ & $909(94)$ & $6.01(0.46)$ \\
\hline thrust law $f\left(. \mid \theta^{*}\right)$ & $m_{\text {estimated }}$ & $9.03(0.38)$ & - & $824(84)$ & $5.83(0.5)$ \\
\hline
\end{tabular}

(a) Case study 1, all the trajectories and observed ESF (A320 climbs departing from Paris).

\begin{tabular}{|c|c||c|c||c|c|}
\hline Thrust/power law & Mass & $e_{k}^{(\text {pred })}$ & p-value & $\Delta H_{p}$ & $\Delta V_{a}$ \\
\hline$B A D A_{\text {max }}$ climb & $m_{B A D A}$ & $9.51(0.25)$ & $1.18 \mathrm{e}-155$ & $1515(58)$ & $4.34(0.23)$ \\
\hline$B A D A_{\text {max }}$ climb & $m_{\text {opt }}$ & $9.57(0.21)$ & $1.29 \mathrm{e}-160$ & $1487(44)$ & $4.04(0.22)$ \\
\hline$B A D A_{\text {max }}$ climb & $m_{\text {estimated }}$ & $7.78(0.32)$ & $3.63 \mathrm{e}-63$ & $1049(80)$ & $4.1(0.14)$ \\
\hline$B A D A_{\text {reduced }}$ & $m_{B A D A}$ & $10.1(0.34)$ & $2.68 \mathrm{e}-172$ & $1708(78)$ & $4.99(0.24)$ \\
\hline$B A D A_{\text {reduced }}$ & $m_{\text {opt }}$ & $9.51(0.22)$ & $5.63 \mathrm{e}-153$ & $1490(45)$ & $4.05(0.23)$ \\
\hline$B A D A_{\text {reduced }}$ & $m_{\text {estimated }}$ & $7.87(0.37)$ & $9.91 \mathrm{e}-64$ & $1040(81)$ & $3.9(0.14)$ \\
\hline thrust law $f\left(. \mid \theta^{*}\right)$ & $m_{\text {estimated }}$ & $7.41(0.3)$ & - & $885(74)$ & $3.62(0.14)$ \\
\hline
\end{tabular}

(b) Case study 2, CAS/Mach trajectories and computed ESF (A320 climbs departing from Paris).

Table 3: Mean values (and standard deviations, within brackets) of the Root Mean Square Errors for $e_{k}^{(\text {pred })}, \Delta H_{p}$ and $\Delta V_{a}$. P-values for the Wilcoxon signed-rank test on $e_{k}^{(\text {pred })}$, when comparing with the last method. 
with $m_{k}^{*}$ the mass of aircraft $k$ estimated on the $n$ past and current points $(n=11$ in our experiments). $c_{k, i}^{(\text {pred })}$ is the thrust setting coefficient at the $i^{\text {th }}$ predicted point, and $Q\left(x_{k, i}^{(o b s)}\right)$ the observed energy rate and wind effect at the $i^{t h}$ actual point:

$$
\begin{aligned}
& c_{k, i}^{(\text {pred })}=f\left(x_{k, i}^{(\text {pred })} \mid \theta^{*}\right) \\
& Q\left(x_{k, i}^{(o b s)}\right)=\left[V_{a} \frac{d V_{a}}{d t}+g_{0}\left(\frac{T}{T-\Delta T}\right) \frac{d H_{p}}{d t}+\frac{d \vec{W}}{d t} \cdot \overrightarrow{V_{a}}\right]_{k, i}
\end{aligned}
$$

In the above equation (31a), $f\left(. \mid \theta^{*}\right)$ is the thrust law learned on the training set, and $x_{k, i}^{(\text {pred })}$ is the vector of state variables (position, velocity, acceleration, etc.) predicted for the $i^{\text {th }}$ point of predicted trajectory $k$.

For a set of trajectories, the overall error on the specific power prediction is assessed by computing the root mean square error (RMSE) of $e_{k}^{(p r e d)}$. This RMSE is computed for the seven chosen combinations of mass and thrust law (see section 5.4), and for the two datasets of the case studies presented in section 5.3 For each fold of the cross-validation, the error is measured over the validation set only, using the thrust law learned on the training set.

The mean values and standard deviations (within brackets) of $R M S E\left(e_{k}^{(\text {pred })}\right)$ are presented in tables $3 \mathrm{a}$ and $3 \mathrm{~b}$, together with the p-values of a Wilcoxon signed-rank paired test comparing our method (last row) to each of the BADA methods. The same table also gives the mean values and standard deviations of the RMSE of $\Delta H_{p}$ and $\Delta V_{a}$, the altitude and velocity errors, respectively, at the last point of the predicted trajectory (10 minutes ahead).

We see on tables $3 \mathrm{a}$ and $3 \mathrm{~b}$ that the methods using the estimated mass $m_{\text {estimated }}$ have lower root mean square errors when compared with the ones using constant masses $\left(m_{B A D A}\right.$ or $\left.m_{O p t}\right)$. So, whatever the chosen thrust/power profile, estimating an individual mass for each aircraft is better than using a common mass, even when this single mass is optimized for the given prediction purpose.

Let us remind that the aim of case study 1 (all trajectories and observed ESF) is to compare the prediction performance on the energy rate only, assuming that the Energy Share Factor is perfectly known. So, in table 3a, we are mostly interested in comparing the errors on $e_{k}^{(p r e d)}$. The errors on $\Delta H_{p}$ and $\Delta V_{a}$ are given as indications. They are not representative of what could actually be obtained in an operational context, when using an $E S F$ law that can differ from the observed $E S F$.

The case study 2 (CAS/Mach trajectories and computed ESF), on the contrary, is more representative of what could be obtained in a real context, assuming the target CAS and Mach number to be known (i.e. transmitted by the aircraft via datalink). We use the computed values of the Energy Share Factor that correspond to the target CAS and Mach number.

Our method combining the estimated mass $m_{\text {estimated }}$ with the adjusted thrust law $f\left(. \mid \theta^{*}\right)$ performs best among the seven tested methods. The statistical significance of 
our results is assessed by performing a Wilcoxon signed-rank tes 5 This choice is relevant because all methods are evaluated on a same dataset (split in ten folds), so we have paired samples: we compare two predictions made with two different methods, for a same flight. Using a directional test, the null hypothesis $\left(H_{0}\right)$ is that the median of the difference between the two predictions (with our method and the other) is positive, meaning that our method performs equally or worse than the other. The alternative hypothesis $\left(H_{1}\right)$ is that the median of the difference is strictly negative, meaning a better performance of our method. The fourth column of tables $3 \mathrm{a}$ and $3 \mathrm{~b}$ shows the $\mathrm{p}$-values ${ }^{6}$ for this test. Comparing with the standard threshold of 0.05 for a $95 \%$ confidence interval, we can reject $H_{0}$ and accept $H_{1}$ as true in all cases.

Let us now look at the altitude prediction, focusing on case study 2 (table $3 \mathrm{~b}$ ). Looking at the altitude error $\Delta H_{p}$ in table 3b we see that our method (last row of the table) reduces the root mean square error by nearly $40 \%$ when compared with the standard BADA maximum climb with the reference mass $m_{B A D A}$ (first row). When comparing with the BADA reduced climb power (third row), the altitude error is reduced by nearly $50 \%$.

\subsection{Altitude Prediction Errors at Various Future Times}

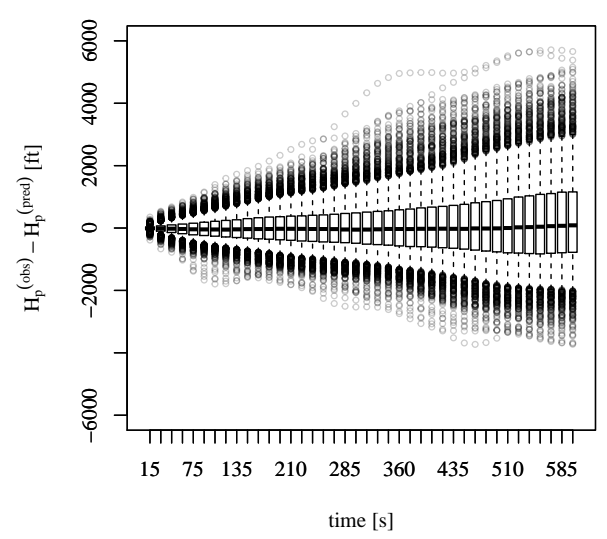

(a) With $B A D A_{\max }$ climb and $m_{B A D A}$.

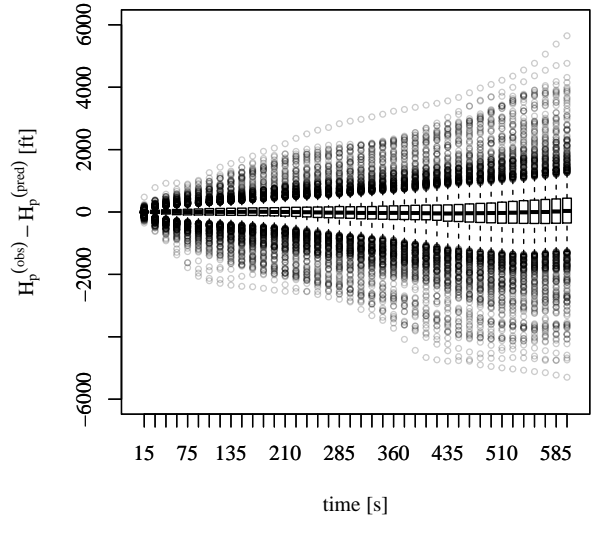

(b) With the inferred mass $m_{\text {estimated }}$ and learned thrust $f\left(. \mid \theta^{*}\right)$.

Figure 6: Altitude errors (case study 2) for future times from $15 \mathrm{~s}$ to $10 \mathrm{mn}$.

Figure 6 shows the boxplots of the altitude errors at various future times, from the first predicted point 15 seconds ahead to the last one 10 minutes ahead, using the

\footnotetext{
${ }^{5}$ We have used the wilcox. test provided by the R environnment, with the paired option.

${ }^{6}$ The p-value is the probability to be wrong when accepting $H_{1}$ and rejecting $H_{0}$.
} 
standard BADA maximum climb (left figure) or our method (right figure). For each look-ahead time in figure 6, the central box represents the $0.25 / 0.75$ interquartile distance, and the whiskers are defined so that the lower one leaves $5 \%$ of the data below it, and the upper one leaves $5 \%$ above.

We can see that the boxes and whiskers are much narrower and better centered around zero with our method than whith the standard BADA method. When using the inferred mass and learned thrust law, the altitude prediction is statistically better at all predicted times.

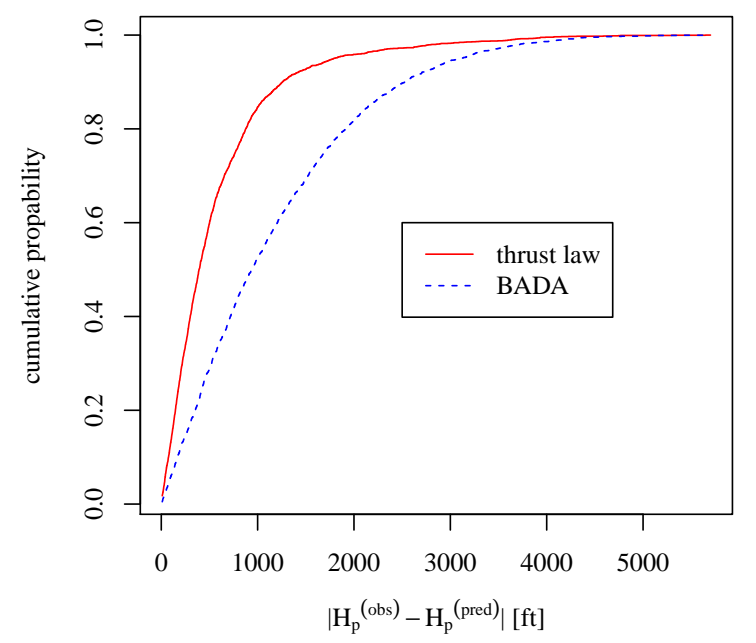

Figure 7: Empirical cumulative distribution of the altitude errors 10 minutes ahead, for the BADA model (blue dotted curve) and our method (red solid curve).

The kurtosis exces: $7^{7}$ for the results of our method is 6.21 , indicating that this distribution is more fat-tailed than the normal one. The distribution obtained with the BADA method, with a kurtosis excess of 0.10 , is much closer to the normal distribution. Nevertheless, our method remains significantly more accurate than BADA, as long as one does not require a confidence interval very close to $100 \%$ : As can be seen on figure 7 , the empirical cumulative distribution function of our method is above the BADA one. Thus, when looking for a centered interval containing $x \%$ of the data, our method will always give a smaller interval than the BADA method.

As can be seen on figure 6, the error range of the predictions falling outside the central cone delimited by the whiskers remains important: Even if the overall performance of our method is better than the BADA method, it could still be improved for a

\footnotetext{
${ }^{7}$ Computed using kurtosis ( . ., type $=1$ ) of the R package 'e1071'
} 
number of specific flights. This suggests that learning several thrust laws for identified classes of climbs, instead of only one for all climbs, might improve the results again.

\subsection{Assessing the Quality of Prediction from the Error on the Past Trajectory}

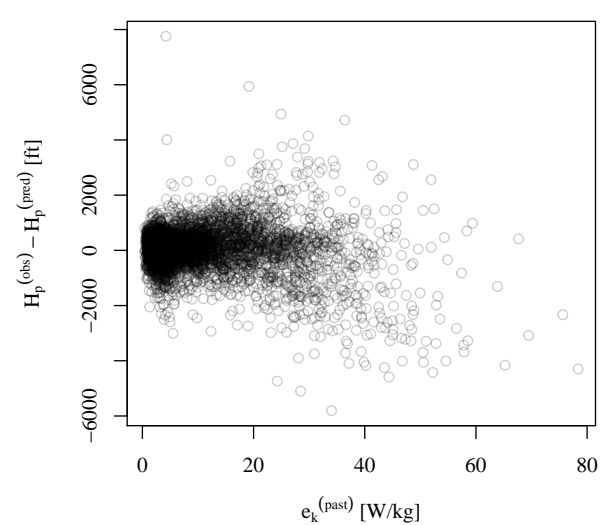

(a) Case study $1, \Delta H_{p}$ against $e_{k}^{(\text {past })}$.

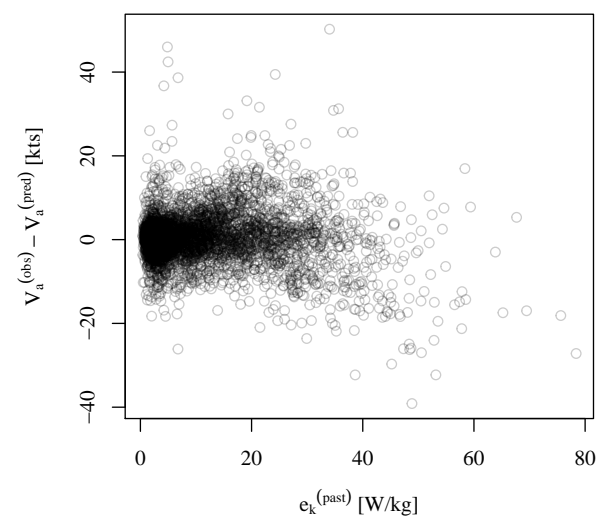

(c) Case study $1, \Delta V_{a}$ against $e_{k}^{(\text {past })}$.

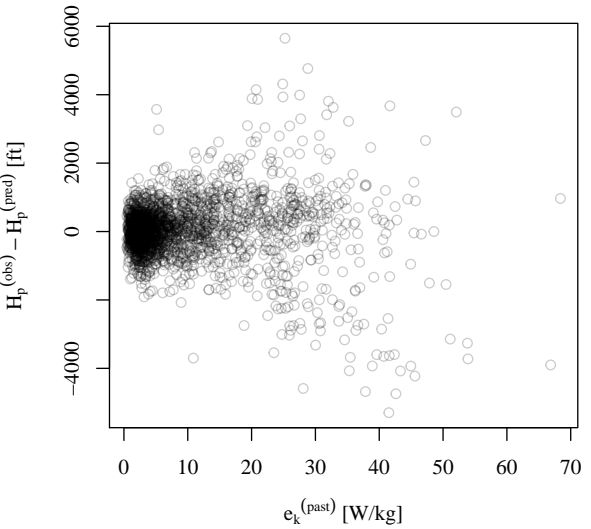

(b) Case study 2, $\Delta H_{p}$ against $e_{k}^{(\text {past })}$.

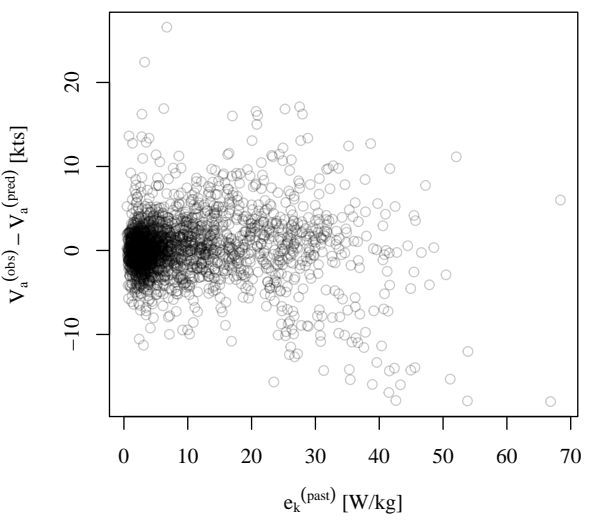

(d) Case study 2, $\Delta V_{a}$ against $e_{k}^{(\text {past })}$.

Figure 8: Relationship between $e_{k}^{(\text {past })}$ and the altitude and velocity errors at the last point of the prediction.

When using the prediction model in an operational context, the future trajectory is yet unknown, and $e_{k}^{(\text {pred) }}$ is not available in real time. However, the model error on the 
$n$ past and current points can be computed, and might give us some clues on the quality of the prediction on $\Delta H_{p}$ and $\Delta V_{a}$.

The expression of $e_{k}^{(\text {past })}$ is similar to the prediction error $e_{k}^{(\text {pred })}$, except that the thrust coefficients are computed using the observed values $x_{k, i}^{(o b s)}$ of the past $n$ points instead of the predicted values $x_{k, i}^{(p r e d)}$ of the $p$ future points:

$$
e_{k}^{(\text {past })}=\sqrt{\frac{1}{n} \sum_{i=1}^{n}(\underbrace{\frac{\operatorname{Power}\left(m_{k}^{*}, c_{k, i}^{(o b s)}\right)}{m_{k}^{*}}}_{\text {computed }}-\underbrace{Q\left(x_{k, i}^{(o b s)}\right)}_{\text {observed at point } i})^{2}}
$$

Figure 8 plots the altitude error $\Delta H_{p}$ and velocity error $\Delta V_{a}$ against $e_{k}^{(\text {past })}$. We can observe a higher dispersion of the plots on the right-hand side of the figures. Globally, we have higher errors on the altitude and velocity when the model error on the past trajectory points $e_{k}^{(\text {past })}$ is high. Notice that this is not always true, however: There are a few trajectories with low errors on $e_{k}^{(p a s t)}$ that exhibit high errors on the altitude and velocity. Trajectories with low model error on the past points are just more likely to have low errors on the predicted altitude and velocity.

As an illustration, let us focus on the trajectories of case study 2 (CAS/Mach trajectories and computed ESF). On these trajectories, the median of $e_{k}^{(\text {past })}$ is $6.6 \mathrm{~W} / \mathrm{kg}$. If we compute the root mean square error of $\Delta H_{p}$ on the trajectories for which $e_{k}^{(\text {past })}$ is inferior to the median, we obtain $532 \mathrm{ft}$. Similarly, if we compute the RMSE on the trajectories with $e_{k}^{\text {(past) }}$ superior or equal to the median, we obtain $1044 \mathrm{ft}$.

To conclude this section, we have an empirical assessment of the quality of the prediction for each individual trajectory simply by considering the model error on the past points. If the model performs poorly on the past points, it is more likely to provide poor predictions on the future points as well.

\section{Conclusion and future work}

To conclude, we propose to improve the trajectory prediction accuracy performed by ground-based systems by inferring the mass and thrust law from observed data. These two parameters, mass and thrust, are crucial in the trajectory prediction of climbing aircraft. The aircraft mass is estimated from a few points of the past trajectory and the thrust law is learned from from a training set of trajectory records. Although they might not be close to the actual mass and thrust law of each individual aircraft, the modeled mass and learned thrust are designed so as to improve the accuracy of the modeled energy rate.

The proposed mass estimation method takes advantage of the fact that the specific power is a polynomial function of the mass when using the BADA model of the drag and maximum climb thrust. Other models of the forces, more precise than BADA, might be compliant with our approach. For instance, the Enhanced Jet Performance Model ([22]) seems to be compliant with our analytical method. 
Our method highly improves the trajectory prediction accuracy when compared with BADA model-based methods that use a single common mass per aircraft type or a standard thrust profile. When tested on real data, for a single aircraft type as a proof of concept, it improves the altitude prediction up to 40 to 50 percent. We also show that the model error on the past trajectory can provide an empirical assessment of the future prediction's quality. From an operational point of view, the enhancement of the altitude prediction accuracy would certainly benefit to the air traffic controllers in the vertical separation task, and give more accurate inputs to the automated tools for conflict detection and resolution.

In future works, it could be interesting to try our method on Mode-S radar data, which is more accurate than Mode-C radar data, and for which the calibrated airspeed and Mach number of the equipped aircraft is available. In addition, we intend to compare our analytical mass estimation method with the adaptive method proposed in [13] on our set of real data.

There are several ways in which our method might be improved. For example, we might try to identify classes of trajectories from the historical data, and then learn several thrust laws (one per class) instead of only one. When predicting a new trajectory, the past points would then be used to select the most relevant thrust law. We could also study different parametrized functions modeling the thrust law: other functions, such as neural networks, might give better results than the simple polynomial functions used in the current paper. Finally, we also plan to compare our model-based approach that uses learned parameters with standard Machine Learning techniques (e.g. Neural Net-

works, Random forests, etc.) that would use the estimated mass and past observations as inputs.

\section{References}

[1] SESAR Consortium. Milestone Deliverable D3: The ATM Target Concept. Technical report, 2007.

[2] H. Swenson, R. Barhydt, and M. Landis. Next Generation Air Transportation System (NGATS) Air Traffic Management (ATM)-Airspace Project. Technical report, National Aeronautics and Space Administration, 2006.

[3] X. Prats, V. Puig, J. Quevedo, and F. Nejjari. Multi-objective optimisation for aircraft departure trajectories minimising noise annoyance. Transportation Research Part C, 18(6):975-989, 2010.

[4] G. Chaloulos, E. Crück, and J. Lygeros. A simulation based study of subliminal control for air traffic management. Transportation Research Part C, 18(6):963974, 2010.

[5] N. Durand, J.M. Alliot, and J. Noailles. Automatic aircraft conflict resolution using genetic algorithms. In Proceedings of the Symposium on Applied Computing, Philadelphia. ACM, 1996. 
[6] F. Drogoul, P. Averty, and R. Weber. Erasmus strategic deconfliction to benefit sesar. In Proceedings of the 8th USA/Europe Air Traffic Management R\&D Seminar, June-July 2009.

[7] C. Vanaret, D. Gianazza, N. Durand, and J.B. Gotteland. Benchmarking conflict resolution algorithms (regular paper). In International Conference on Research in Air Transportation (ICRAT), Berkeley, California, 22/05/12-25/05/12, page (on line), http://www.icrat.org, may 2012. ICRAT.

[8] I. Lymperopoulos, J. Lygeros, and A. Lecchini Visintini. Model Based Aircraft Trajectory Prediction during Takeoff. In AIAA Guidance, Navigation and Control Conference and Exhibit, Keystone, Colorado, August 2006.

[9] A. Hadjaz, , G. Marceau, P. Savéant, and M. Schoenauer. Online learning for ground trajectory prediction. In Proceedings of the SESAR Innovation Days (2012). EUROCONTROL, 2012.

[10] G. L. Slater. Adaptive improvement of aircraft climb performance for air traffic control applications. In Proceedings of the 2002 IEEE International Symposium on Intelligent Control. IEEE conference publications, October 2002.

[11] A.W. Warren and Y.S. Ebrahimi. Vertical path trajectory prediction for next generation atm. In Digital Avionics Systems Conference, 1998. Proceedings., 17th DASC. The AIAA/IEEE/SAE, volume 2, pages F11/1 -F11/8 vol.2, oct-7 nov 1998.

[12] A.W. Warren. Trajectory prediction concepts for next generation air traffic management. In 3rd USA/Europe ATM R\&D Seminar, June 2000.

[13] C. Schultz, D. Thipphavong, and H. Erzberger. Adaptive trajectory prediction algorithm for climbing flights. In AIAA Guidance, Navigation, and Control (GNC) Conference, August 2012.

[14] R. Alligier, D. Gianazza, and N. Durand. Energy Rate Prediction Using an Equivalent Thrust Setting Profile (regular paper). In International Conference on Research in Air Transportation (ICRAT), Berkeley, California, 22/05/12-25/05/12, page (on line), http://www.icrat.org, may 2012. ICRAT.

[15] R. Alligier, D. Gianazza, and N. Durand. Ground-based estimation of aircraft mass, adaptive vs. least squares method. In Proceedings of the 10th USA/Europe Air Traffic Management $R$ \& D Seminar, 2013.

[16] R. Slattery and Y. Zhao. Trajectory synthesis for air traffic automation. Journal of Guidance, Control and Dynamics, 20(2):232-238, 1997.

[17] A. Nuic. User manual for base of aircarft data (bada) rev.3.9. Technical report, EUROCONTROL, 2011. 
[18] A. Nuic, C. Poinsot, M. Iagaru, E. Gallo, F.A. Navarro, and C. Querejeta. Advanced aircraft performance modeling for atm: Enhancements to the bada model. In Proceedings of the 24th IEEE/AIAA Digital Avionics Systems Conference (DASC), Oct-Nov 2005.

[19] D. Poles, A. Nuic, and V. Mouillet. Advanced aircraft performance modeling for atm: Analysis of bada model capabilities. In Proceedings of the 29th IEEE/AIAA Digital Avionics Systems Conference (DASC), Oct 2010.

[20] J. Nocedal and S.J. Wright. Numerical Optimization. Springer series in operations research and financial engineering. Springer Science+Business Media, LLC., 2006.

[21] Icao annex 10 on the convention on international civil aviation, vol iv, chaptersection: 3.1.1.7.12.2.3. Technical report, International Civil Aviation Organization.

[22] M. Kaiser, M. Schultz, and H. Fricke. Enhanced jet performance model for high precision $4 \mathrm{~d}$ flight path prediction. In Proceedings of the 1st International Conference on Application and Theory of Automation in Command and Control Systems, ATACCS '11, pages 33-40, Toulouse, France, France, 2011. IRIT Press. 